This document is the accepted manuscript version of the following article:

Anthis, A. H. C., Tsolaki, E., Didierlaurent, L., Staubli, S., Zboray, R., Neels, A., ... Herrmann, I. K. (2019). Nano-analytical characterization of endogenous minerals in healthy placental tissue: mineral distribution, composition and ultrastructure. Analyst, 144, 6850-6857. https://doi.org/10.1039/C9AN01312A

\title{
Nano-analytical Characterization of Endogenous Minerals in Healthy Pla- cental Tissue: Mineral Distribution, Composition and Ultrastructure
}

Alexandre H.C. Anthis ${ }^{a, b}$, Elena Tsolakic, Louis Didierlaurent ${ }^{a}$, Samuel Staublia ${ }^{a}$, Robert Zboray ${ }^{d}$, Antonia Neels $^{d}$, Dörthe Dietrich ${ }^{\mathrm{e}}$, Pius Manser ${ }^{\mathrm{a}}$, Lotus May Desbiolles ${ }^{f}$, Sebastian Leschka ${ }^{f}$, Simon Wildermuth ${ }^{f}$, Sandro Lehner ${ }^{\mathrm{g}}$, Pascale Chavatte-Palmer ${ }^{h}$, Wolfram Jochumi, Peter Wick ${ }^{a}$, Alex Dommannn ${ }^{\mathrm{a}, \mathrm{d}}$, Tina Bürki-Turnherr ${ }^{\mathrm{a}}$, Tina Fischer ${ }^{\mathrm{j}}$, René Hornung ${ }^{j}$, Sergio Bertazzoc ${ }^{c}$ Inge K. Herrmann ${ }^{a, b *}$

\footnotetext{
aLaboratory for Particles Biology Interactions, Department Materials Meet Life, Swiss Federal Laboratories for Materials Science and Technology (Empa), Lerchenfeldstrasse 5, CH-9014, St. Gallen, Switzerland.

bNanoparticle Systems Engineering Laboratory, Institute of Process Engineering (IPE), Department of Mechanical and Process Engineering (D-MAVT), ETH Zurich, Sonneggstrasse 3, CH-8092, Zurich, Switzerland.

'Department of Medical Physics \& Biomedical Engineering, University College London, London WC1E 6BT, UK. dCenter for X-ray Analytics, Department Materials Meet Life, Swiss Federal Laboratories for Materials Science and Technology (Empa), Überlandstrasse 129, CH-8900, Dübendorf, Switzerland.

eWestfälische Wilhelms-Universität Münster, Institute of Inorganic and Analytical Chemistry, Corrensstraße 30, D48149 Münster, Germany

fDivision of Radiology and Nuclear Medicine, Cantonal Hospital St. Gallen (KSSG), Rorschacherstrasse 95, CH-9007, St. Gallen, Switzerland.

gLaboratory for Advanced Fibers and Laboratory for Biointerfaces, Department Materials Meet Life, Swiss Federal Laboratories for Materials Science and Technology (Empa), Lerchenfeldstrasse 5, CH-9014, St. Gallen, Switzerland. hUMR BDR, INRA, ENVA, Université Paris Saclay, F-78350, Jouy en Josas, France.

institute of Pathology, Cantonal Hospital St. Gallen (KSSG), Rorschacherstrasse 95, CH-9007, St. Gallen, Switzerland.

jDepartment of Gynaecology, Cantonal Hospital St. Gallen (KSSG), Rorschacherstrasse 95, CH-9007, St. Gallen, Switzerland.

+ A.H.C.A and E.T. contributed equally as first authors. S.B. and I.K.H. contributed equally as senior authors
}

Despite its crucial role, the placenta is the least understood human organ. Recent clinical studies indicate a direct association between placental calcification and maternal and offspring health. This study reveals distinct characteristics of minerals formed during gestational ageing using cutting-edge nano-analytical characterization and paves the way for investigations focused on the identification of potential markers for disease risks in a clinical setting based on atypical placental mineral fingerprints.

Similar to calcification in the heart, ${ }^{1,2}$ placental calcification ${ }^{3,4}$ is a phenomenon that has been known for decades, yet remains poorly understood. ${ }^{5}$ While it is well-known that the extent and tissue-specific patterns of vascular calcification are predictors of cardiovascular morbidity and mortality, placental calcifications are scarcely researched. ${ }^{5}$ Despite its fundamental role, ${ }^{6}$ it remains to be the least understood human organ. ${ }^{7}$ The placenta mediates communication between two circulatory systems and dynamically adapts its morphology and function for optimal fetal growth in order to cope with suboptimal conditions, and hence contains a wealth of healthrelevant information. ${ }^{8}$ There is a growing body of evidence that underlines the importance of placental development in the lifelong health of both mother and offspring. ${ }^{5}$ While the correlation between fetal outcome and placental calcification has been known for decades, ${ }^{9,} 10$ recent epidemiological and clinical studies suggest that there is an association between placental insufficiencies (e.g., caused by excessive calcification) and vulnerability 
for adult disease, thus directly linking placental characteristics to adult health. ${ }^{11}$ Placental phenotypic traits have been associated with diseases, including heart disease, hypertension, insulin resistance, asthma, cancers, as well as premature death. ${ }^{12}$ Complementing the ongoing effort focused on biochemical aspects, ${ }^{5}$ the understanding of the mineral characteristics and formation mechanisms of placental calcification and its wider implications are imperative to the development of preventive and curative strategies. Placental calcification may share etiologic traits with other forms of ectopic calcification. Ectopic calcification may form by metastatic, dystrophic, or physiologic mechanisms. Studies found activation of programmed cell death pathways and presence of necrotic tissue suggesting that dystrophic calcification may at least contribute to placental calcification. ${ }^{5}$ While it remains largely unexamined whether placental calcification involves physiological calcification mechanisms, it has been demonstrated that placenta cells have osteogenic potential, which may be different for cells from different anatomical parts from the placenta. ${ }^{13}$ Other studies found bone morphogenetic proteins were not directly related to placental calcification and that the chemical composition of the mineral was suggestive of rapid formation in supersaturated conditions, which pinpoints towards a metastatic mechanism. ${ }^{14}$

Most of the placenta calcification research has focused on clinical sonographic imaging ${ }^{9,15}$ and histological tissue analysis with poor sensitivity for mineral characteristics. Calcium phosphate-based minerals have been identified in placental tissues by X-ray techniques, mostly in the basal plate facing the endometrium but also in the tunica media and the intima of the umbilical cord. ${ }^{3,4}$ Recent improvements in instrumentation technology and sample preparation have rendered key materials science characterization techniques suitable for the analysis of complex biological and biomimetic systems, ${ }^{16-18}$ hence providing access to a plethora of ultrastructural information at unprecedented resolution. By employing nano-analytical materials characterization, it has recently been discovered that calcific lesions in cardiovascular mineralization are not entirely composed of bone, but contain nano- and micro-sized highly crystalline magnesium-containing calcium phosphate particles. ${ }^{18}$ Similar particles have also been identified in mineralized osteocyte lacunae. ${ }^{19,20}$

Here, we investigate placental calcification by examining fresh human placental tissue samples by materials characterization techniques across scale. We explore the localization and the nano-structural characteristics of mineral deposits in placental tissues with nanoscale resolution by employing cutting-edge materials science techniques. We identify several distinctly different forms of mineral in placental tissue and show considerable intersubject variability in mineral content even in placentas from clinically uncomplicated pregnancies. By including clinically established CT and histological analysis in the multiscale analytical characterization cascade, we put the findings into context and pave the way for a refined ultrastructural analysis of mineral deposits in large clinical studies.

We analysed thirty placentas from healthy mothers who delivered between 37 and 40 weeks of gestation. Samples were taken from different tissue regions as illustrated in Figure 1a. First, the total mineral content was determined by elemental analysis following tissue digestion. For the analysis of the mineral distribution, the placental tissue samples were collected from central and peripheral tissues, and from the maternal and the fetal portion of the placenta. Both calcium and magnesium showed considerable inter-subject variation (range for Ca: 0.6-32 mg per g for the maternal portion and 0.7-76 mg per g for the fetal portion, based on dry weight, Figure 
1b). Differences between the different anatomical regions were minor. No significant difference was found between central (median and Cl95\% for Ca: 1.5 [1.17, 4.43] mg per g // median and Cl95\% for Mg: 0.4 [0.39, 0.48] mg per g) and peripheral (median and Cl95\% for Ca: 1.6 [0.791, 6.809] mg per g // median and Cl95\% for Mg: $0.4[0.37,0.5] \mathrm{mg}$ per $\mathrm{g}$ ) (see also Figure $\mathrm{S} 1, \mathrm{~N}=22$ ). A trend towards slightly higher mineral contents on the maternal (median for Ca: 4.8 [2.8, 14.2] mg per g // median for Mg: 0.48 [0.4, 0.66] mg per g) compared to the fetal portion (median for Ca: 1.4 [-2.2, 19.2] mg per g // median for Mg: $0.5[0.39,0.6] \mathrm{mg}$ per g) of the placenta was found (see also Figure $\mathrm{S} 2, \mathrm{~N}=15$ ). There was a significant correlation between the $\mathrm{Ca}$ and $\mathrm{Mg}$ content (Spearman's rho: $0.4, p<0.01$ ). The total calcium measurements are in agreement with a study by de Moraes et al. ${ }^{21}$ which found median calcium concentrations around $0.7 \mathrm{mg}$ per $\mathrm{g}$ (maternal portion) and $2 \mathrm{mg}$ per $\mathrm{g}$ (fetal portion). Overall, de Moraes et al. found strikingly similar inter-subject variations in total calcium content in placental tissue in both maternal (range: $0.017-32.4 \mathrm{mg}$ per g) and fetal (0.1-64.7 mg per g) portions of the placenta.

While not all the mineral may be present in the precipitated form, the solid fraction was further characterized by multiscale materials characterization techniques. In order to gain insights into the macroscopic distribution of the precipitated fraction of mineral, $\mathrm{X}$-ray tomography scans of entire placentas were performed on a clinical computer tomograph (CT). Figure 1c shows reconstructed images of two representative placentas, one with low and one with high mineral content. In terms of mineral distribution, the larger part of mineral deposits detectable by clinical CT (>1mm) is localized in the peripheral regions of the placenta, and preferentially on the maternal side. The preferential accumulation of mineral on the maternal portion (basal plate) and the villous part of the placenta has been further confirmed by micro-tomographic data with higher spatial resolution (voxel size of $\sim 10$ $\mu \mathrm{m}^{3}$ ) (Figure 1d). The CT results, together with the total mineral content measured by elemental analysis, indicate that the extent of mineralization is of significant variation even in placentas from clinically uncomplicated pregnancies.

Since CT imaging only allows imaging of relatively large mineral structures in the micron-range and gives no access to chemical composition, we performed additional mesoscale analyses by micro-X-ray fluorescence ( $\mu \mathrm{XRF}$ ) mapping and correlated the data to histology data sets. Figure 1e shows $\mu$ XRF maps of a histological cross section of placental tissue. Calcium and phosphorus maps show co-localized accumulation and indicate the presence of calcium phosphate deposits of varying size. Magnesium contents remained below the detection limit. The mineral deposits are also visible in histological analysis where the contained calcium stains positive in Alizarin Red stained sections (Figure 1f).

In order gain insights into the ultrastructure of the mineral deposits, we analysed placental tissue samples from both the fetal and the maternal portion (basal plate) by scanning electron microscopy (SEM). SEM allows characterization of mineral particles at scales inaccessible to optical microscopy and reveals the presence of a wide variety of distinct mineral morphologies (Figure 2). Secondary electron (SE) and backscattering electron (BSE) signals were collected from the same region and Density Dependent Colour SEM (DDC-SEM) images were assembled by assigning the SE image to the green channel and the BSE image to the red channel of a RGB stack. Electron-dense fibre-like structures can be found in the maternal portion of the placenta (Figure 2a) along with 
particle deposits. In addition to the larger blocks of minerals seen in CT, XRF and histology imaging, significantly smaller structures can be identified in scanning electron microscopy. High magnification images show calcified fibres and spherical particles (Figure 2b,c). Energy-dispersive X-ray Spectroscopy (EDXS) indicates that the particle-containing fibre-like structures contain traces of magnesium in addition to calcium. Structures feature compositional and morphological similarities to particles of cellular origin found in mineralized osteocyte lacunae ${ }^{19}$ and in aortic tissues. ${ }^{18}$ In addition to calcified fibres and particles, larger chunks of hydroxyapatite mineral with a poorly defined shape and a size of several tens of microns have been found in the majority of the samples investigated (Figure 3a). In the villous regions, mineral deposits are generally smaller and more diffuse (Figure 3b). EDXS analysis of several of the imaged structures indicates that the predominant form of mineral contains calcium and phosphorous (see also Figure S3). In agreement with the CT and elemental analysis measurements, mineral deposits are generally sparser on the fetal side. Relatively large $(>10 \mu \mathrm{m})$ needle-like structures are observed in several of the samples (Figure 3c). Raman spectroscopic analysis performed on the samples from the different anatomical regions confirms the presence of calcium phosphate mineral (Raman shift of $960 \mathrm{~cm}^{-1}$, Figure 3d) in the majority of the samples investigated. In rare cases, and in agreement with the EDXS measurements, magnesium-containing apatite has been found as indicated by a Raman shift of the phosphate peak to $970 \mathrm{~cm}^{-}$ ${ }^{1} .{ }^{17} \mathrm{X}$-ray diffraction analysis of mineral isolated from tissue suggest the presence of a significant crystalline fraction of hexagonal hydroxyapatite (no evidence for $\mathrm{F}$ or $\mathrm{Cl}$ was found in EDXS), ${ }^{22}$ in addition to a background signal originating from amorphous constituents (Figure 3e). The characteristics of the larger chunks consisting of hexagonal hydroxyapatite are in line with minerals found in dystrophic calcification ${ }^{23}$ observed in degenerated and necrotic tissue and may thus be a mere consequence of tissue aging. The other, more defined structures, including the needles and the small spherical particles pinpoint to at least a second, if not third underlying mechanism of calcification different from the dystrophic one. ${ }^{14,24}$ Additionally, the deviations in composition suggests a mineralization process unlike regular bone mineralization. ${ }^{24}$ Surprisingly, while calcifications were found in all human placentas to a variable degree, no significant placenta calcification was found in investigated animal placentas, neither in carnivores/omnivores (4 cats, 2 dogs) nor herbivores (2 rabbits, 2 horses).

\section{Conclusions}

Taken together, this study shows that the extent of placental calcification as well as the type of mineral found in placentas obtained following the delivery is very diverse. Clinically, it is poorly understood why some cases of excessive placental calcifications have negative impact on fetal outcome while others have not. Based on the ultrastructural examinations proposed here, new clinical studies can now be initiated to dissect dystrophic from other forms of mineralization with potential pathological relevance. More excitingly, clinical studies investigating connections of placental calcification with maternal and offspring health can now integrate such material characterizations to complement the biochemical and clinical data. Such new ultrastructural insights provided by materials characterization type of analyses, combined with the growing body of data obtained by traditional biochemical analysis have three important implications. i) Clinical studies on factors influencing preterm placental calcification, including hypertension, smoking and environmental factors can now be performed including 
quantitative mineral characterization data. ii) Mechanistic studies based on compositional and structural similarities of placental minerals and minerals in other tissues may be initiated to identify potential connections between calcific deposits in different tissues.

Additionally, information on tissue-specific growth kinetics may be extracted. iii) Atypical minerals formed during gestation may now be identified in large clinical studies based on the above advanced mineral characterization cascade and may serve as early markers identifying susceptible individuals at risk ${ }^{12}$ for adult disease.

\section{Experimental Section}

Placenta. The project was approved by the local ethics committee (EKSG 10/078) and performed in accordance with the principles of the Declaration of Helsinki. Human placentas were obtained from uncomplicated term pregnancies after caesarean section from the Kantonsspital St. Gallen (KSSG). Written informed consent was obtained prior to delivery.

$X$-ray tomography. The entire placentas were transported to the computer tomography unit at the radiology department at the local hospital and scanned within $2 \mathrm{hrs}$ after delivery $(\mathrm{N}=7)$. CT was performed on a clinical scanner (Somatom Force, Siemens Healthineers, Forchheim, Germany) in the single-source mode with the use of the following parameters: tube voltage, $120 \mathrm{kVp}$; tube current-exposure time product, $380 \mathrm{mAs}$; collimation, $192 \times 0.6 \mathrm{~mm}$; gantry rotation time, $250 \mathrm{~ms}$; and pitch, 0.9 . The data were reconstructed in $0.2-\mathrm{mm}$ increments at a slice thickness of $0.4 \mathrm{~mm}$, with the use of a medium soft-tissue and a sharp convolution filter. Volumerendering technique was used for colour-coded 3D visualization of the CT dataset.

Sampling. Ten full thickness biopsies with a diameter of $8 \mathrm{~mm}$ were collected from locations indicated in Figure 1 and either flash-frozen or chemically fixed in 4\% paraformaldehyde (from all thirty different placentas, $\mathrm{N}=30$ ).

Micro X-ray tomography. For microCT, the biopsies (four biopsies from two representative placentas, one containing high- and one low-grade calcification) were gradually dehydrated by ethanol and placed in Eppendorf tubes in a saturated ethanol atmosphere. The samples were mounted on a sample holder and scanned on an EasyTom microCT scanner (RxSolutions SAS, Chavanod, France), with a tungsten target operated at $40 \mathrm{kV}$ with an emission current of $90 \mu \mathrm{A}$. The voxel size of the microCT scans was $10 \mu \mathrm{m}$.

Elemental Analysis. For elemental analysis, frozen tissue samples were dehydrated by submersion in ultrapure ethanol for 24 hours and then dried at $40^{\circ} \mathrm{C}$ in a vacuum oven overnight. The samples were then digested using $2.5 \mathrm{~mL}$ of ultrapure $\mathrm{HNO}_{3}$ and $1 \mathrm{~mL}$ of $30 \% \mathrm{H}_{2} \mathrm{O}_{2}$ per sample, in a microwave digestion unit (TURBOWAVE). Samples were analysed on an Agilent Inductively Coupled Plasma Optical Emission Spectrometer (ICP-OES, 5110) based on the sample dry weight.

Histology. For histological analysis, fixed or frozen samples from four representative placentas were sent to SophistoLab, Muttenz, Switzerland for sectioning and staining. Sections were stained with Alizarin Red S to reveal macroscopic calcifications. Alizarin Red S ( $2 \mathrm{~g})$ was dissolved in $100 \mathrm{~mL}$ of distilled water and pH was adjusted to 4.2. Slides were stained with freshly prepared Alizarin Red solution for two minutes. Images were recorded on a 
Zeiss Microscope using 10x and 40x objectives.

$X$-ray Fluorescence Mapping. Unstained tissue sections mounted on glass slides were analyzed by non-destructive $\mu$-XRF measurements performed on a TORNADO M4 X-ray fluorescence spectrometer (Bruker Nano $\mathrm{GmbH}$, Berlin, Germany) according to a previously described protocol. ${ }^{25}$

Raman Spectroscopy. For Raman spectroscopic analysis, samples prepared for SEM were mounted on a glass coverslip and analysed in a WITEC 300alphaR confocal Raman system equipped with a 50× LD objective (NA 0.55) and a $532 \mathrm{~nm}$ laser. For acquisition, the laser power was set to $5 \mathrm{~mW}$ and spectral integration times were set to $5 s$.

Scanning Electron Microscopy. Frozen placenta samples from the thirty different placentas taken from different anatomical locations (fetal, villous or maternal part) were dehydrated using an ethanol gradient and mounted on silver stubs. The samples were sputtered with $5 \mathrm{~nm}$ carbon. A Hitatchi S-3499N, a Carl Zeiss VP and a LEO Gemini 1525 FEGSEM were used for SEM analysis. Secondary electron (SE) and backscattering electron (BSE) signals were collected and density-dependent images (DDC-SEM) were assembled using the RGB stack option in ImageJ. Energy Dispersive X-ray Spectroscopy analysis was carried out using Oxford Instruments EDX detectors. For the analysis, accelerating voltages of $5 \mathrm{kV}$ and $10 \mathrm{kV}$ and a working distance of $10 \mathrm{~mm}$ were used.

Mineral extraction, isolation and analysis by X-ray diffraction. For X-ray diffraction measurements, minerals were isolated from tissue by mechanical digestion followed by collagenase treatment and high-speed centrifugation. The white pellet forming at the bottom was collected, washed with water and ethanol and dried in vacuum prior to analyses. X-ray diffraction patterns have been measured on a Stoe Mark II-Imaging Plate Diffractometer System (Stoe \& Cie, 2015) equipped with a graphite-monochromator. Data collection was performed at $-100^{\circ} \mathrm{C}$ using Mo-K $\alpha$ radiation $(\lambda=0.71 \AA$, beam diameter $0.5 \mathrm{~mm}$ ). Two-dimensional diffraction images (15 min per exposure) were obtained at an image plate distance of $200 \mathrm{~mm}$ with a continued sample rotation. The resolution was $D_{\min }-D_{\max } 24.00$ - $1.04 \AA$ and intensity integration has been performed over the entire image $\left(360^{\circ}\right)$.

Animal placentas. Placentas from different species were obtained from INRA. In the study, four cat placentas (gestational age 35w), two dog placentas (gestational age 42 weeks), two horse placentas (term) and two rabbit placentas (gestational age 28 weeks) were analyzed.

Author contributions: A.H.C.A., E.T. L.D., S.S., and S.L. harvested samples and performed elemental analysis, electron microscopy experiments. R.Z. performed microCT measurements. A.N. Performed XRD measurements. D.D. performed XRF measurements. P.M. aided with sample procurement. L.M.D., S.L. and S.W. performed clinical CT measurements and analysed clinical CT data. P.C.P aided with sample procurement and analysis of animal placentas. W.J. analysed pathology data. P.W., A.D., T.B.T., T.F. and R.H. and all athor authors analysed and interpreted the data. S.B. and I.K.H. supervised the study. I.K.H. wrote the manuscript together with all co-authors.

\section{Conflicts of interest}

There are no conflicts to declare. 


\section{Notes and references}

1. M. Y. Speer and C. M. Giachelli, Cardiovascular Pathology, 2004, 13, 63-70.

2. B. B. Tomazic, Characterization of Mineral Phases in Cardiovascular Calcification, Crc Press Inc, Boca Raton, 1994.

3. V. A. Varma and K. M. Kim, Scan Electron Microsc, 1985, 1567-1572.

4. S. H. Poggi, K. I. Bostrom, L. L. Demer, H. C. Skinner and B. J. Koos, Placenta, 22, 591-596.

5. M. C. Wallingford, C. Benson, N. W. Chavkin, M. T. Chin and M. G. Frasch, Front Physiol, 2018, 9, 10441044.

6. T. Matikainen and J. Laine, Toxicology and Applied Pharmacology, 2005, 207, 544-549.

7. A. E. Guttmacher, Y. T. Maddox and C. Y. Spong, Placenta, 2014, 35, 303-304.

8. A. N. Sferruzzi-Perri, J. S. Higgins, O. R. Vaughan, A. J. Murray and A. L. Fowden, Proceedings of the National Academy of Sciences, 2019, 116, 1621-1626.

9. K. H. Chen, L. R. Chen and Y. H. Lee, Ultrasound in Obstetrics \& Gynecology, 2011, 37, 328-334.

10. K.-H. Chen, K.-M. Seow and L.-R. Chen, Placenta, 2015, 36, 1039-1044.

11. K. L. Thornburg, P. F. O'Tierney and S. Louey, Placenta, 2010, 31, S54-S59.

12. A. N. Sferruzzi-Perri and E. J. Camm, Frontiers in Physiology, 2016, 7.

13. G. Kmiecik, V. Spoldi, A. Silini and O. Parolini, Stem Cell Reviews and Reports, 2015, 11, 570.

14. S. H. Poggi, K. I. Bostrom, L. L. Demer, H. C. Skinner and B. J. Koos, Placenta, 2001, 22, 591-596.

15. S. A. Mastrolia, A. Y. Weintraub, Y. Sciaky-Tamir, D. Tirosh, G. Loverro and R. Hershkovitz, The Journal of Maternal-Fetal \& Neonatal Medicine, 2016, 29, 921-927.

16. A. K. Rajasekharan, R. Bordes, C. Sandström, M. Ekh and M. Andersson, Small, 2017, 13, 1700550.

17. A. Y. F. You, M. S. Bergholt, J.-P. St-Pierre, W. Kit-Anan, I. J. Pence, A. H. Chester, M. H. Yacoub, S. Bertazzo and M. M. Stevens, Science Advances, 2017, 3.

18. S. Bertazzo, E. Gentleman, K. L. Cloyd, A. H. Chester, M. H. Yacoub and M. M. Stevens, Nature Materials, 2013, 12, 576.

19. P. Milovanovic, E. A. Zimmermann, A. vom Scheidt, B. Hoffmann, G. Sarau, T. Yorgan, M. Schweizer, M. Amling, S. Christiansen and B. Busse, Small, 2017, 13, 1602215.

20. F. A. Shah, B. E. J. Lee, J. Tedesco, C. Larsson Wexell, C. Persson, P. Thomsen, K. Grandfield and A. Palmquist, Nano Letters, 2017, 17, 6210-6216.

21. M. L. de Moraes, R. de Faria Barbosa, R. E. Santo, F. da Silva Santos, L. B. de Almeida, E. F. O. de Jesus, F. L. de Carvalho Sardinha and M. d. G. T. do Carmo, Biological Trace Element Research, 2011, 143, 1271-1281.

22. S. V. Dorozhkin and M. Epple, Angewandte Chemie International Edition, 2002, 41, 3130-3146.

23. J. R. Ortlepp, F. Schmitz, V. Mevissen, S. Weiß, J. Huster, R. Dronskowski, G. Langebartels, R. Autschbach, K. Zerres, C. Weber, P. Hanrath and R. Hoffmann, European Heart Journal, 2004, 25, 514-522.

24. S. C. Curtze, M. Kratz, M. Steinert and S. Vogt, Scientific reports, 2016, 6, 23285-23285.

25. L. Veith, D. Dietrich, A. Vennemann, D. Breitenstein, C. Engelhard, U. Karst, M. Sperling, M. Wiemann and B. Hagenhoff, Journal of Analytical Atomic Spectrometry, 2018, 33, 491-501. 
a)

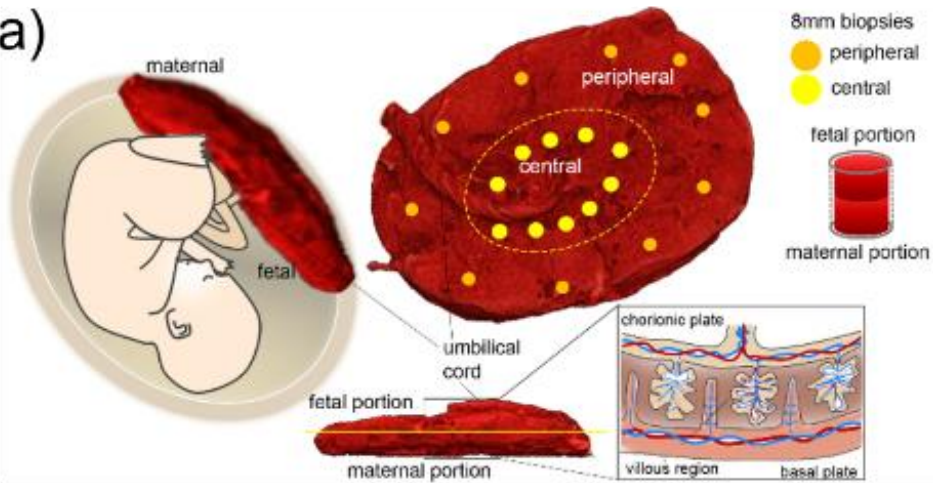

c)

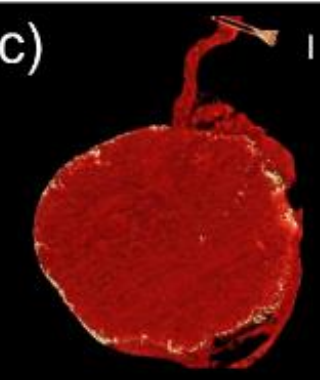

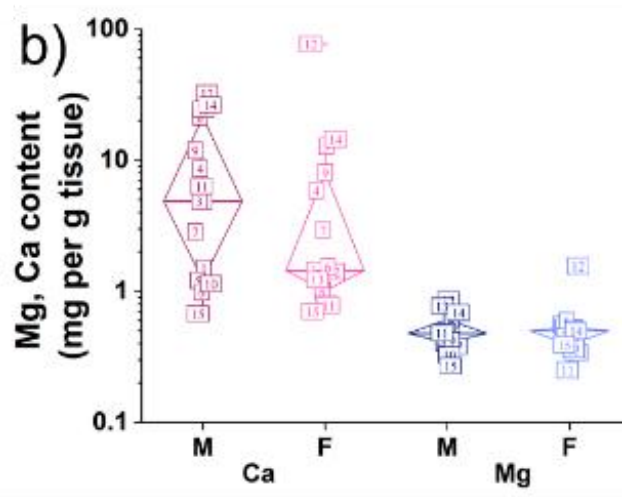

III d)
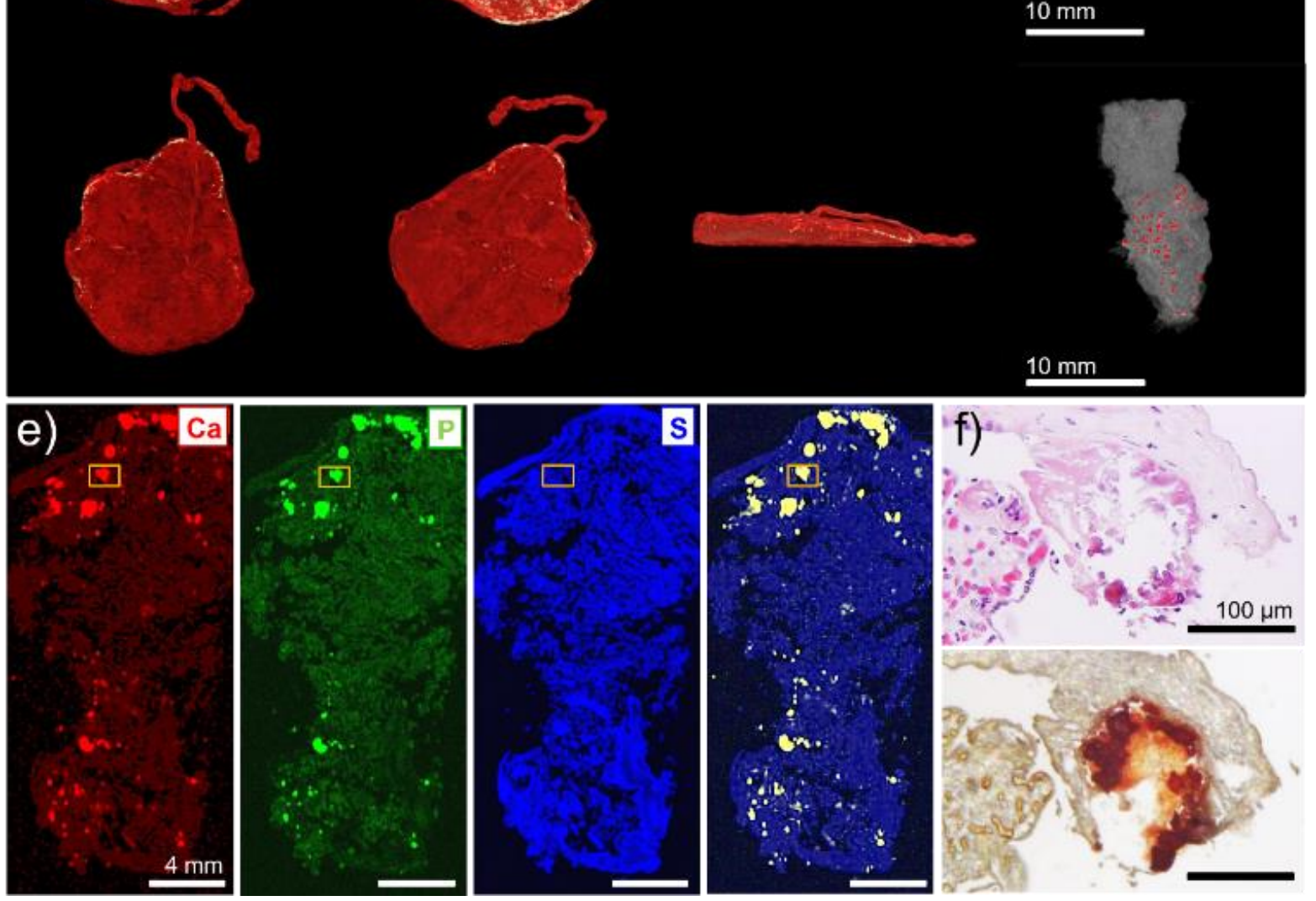

Figure 1: (a) Anatomical structure of the placenta showing the umbilical cord, the chorionic plate and the villous chorion (fetal side), and the basal plate (maternal side). Yellow and orange marks indicate locations of sample collection. (b) Total mineral content of placental samples (dry weight) measured by ICP-OES. Sampling of the maternal and the fetal side. (c) Computer tomography of two representative entire placentas (with high-grade and low-grade calcification). Frontal view of the fetal (I) and the maternal side (II) and lateral view (III). (d) Corresponding micro-computer tomography $(\mu \mathrm{CT})$ of a $8 \mathrm{~mm}$ biopsy punch sample with a voxel size of $10 \mu \mathrm{m}^{3}$ (top: fetal portion). (e) $\mu$-Xray Fluorescence map of a placental cross section (top: fetal part) showing the calcium, phosphorus and sulfur distribution and the overlay. Mg was not detectable by XRF. (f) Corresponding H\&E staining and Alizarin Red S staining (calcium) of placental tissue of the region indicated in (e). 

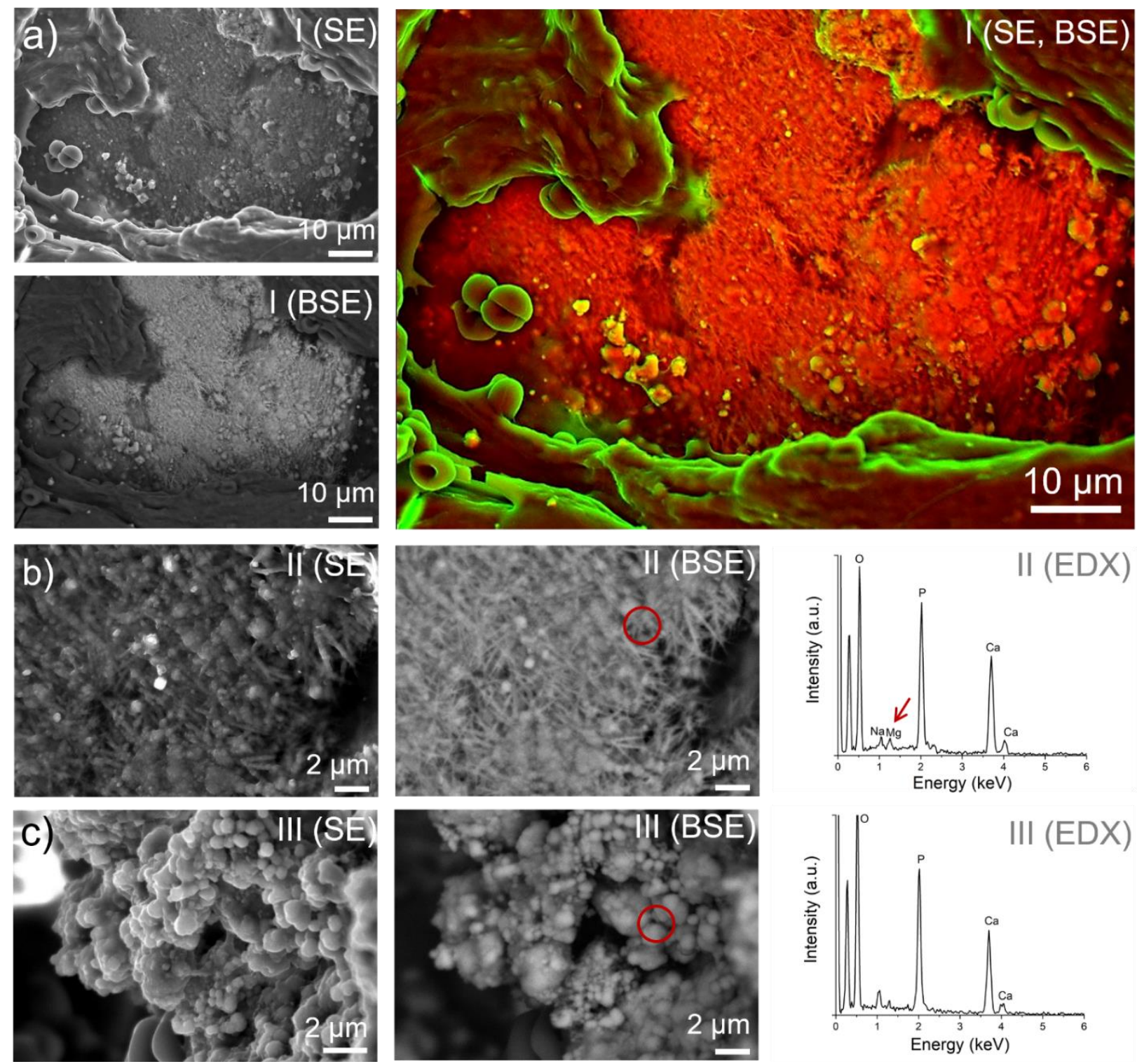

Figure 2: (a) Scanning electron micrographs mineral structures identified in placental tissue (maternal side). Density dependent color scanning electron micrographs (DDC-SEM) consisting of an overlay of secondary electron (I, SE, green) and back scattered electron (I, BSE, red) signals illustrating dense minerals surrounded by soft tissue. (b) Fibrous region imaged by SE and BSE, and corresponding EDXS. (c) Particle-rich region imaged by SE and BSE, and corresponding EDX. EDX spectra indicate the presence of $\mathrm{Ca}, \mathrm{P}$ as well as traces of $\mathrm{Mg}$. No evidence for $\mathrm{F}$ or $\mathrm{Cl}$ was found in EDXS. 
a) Fetal side
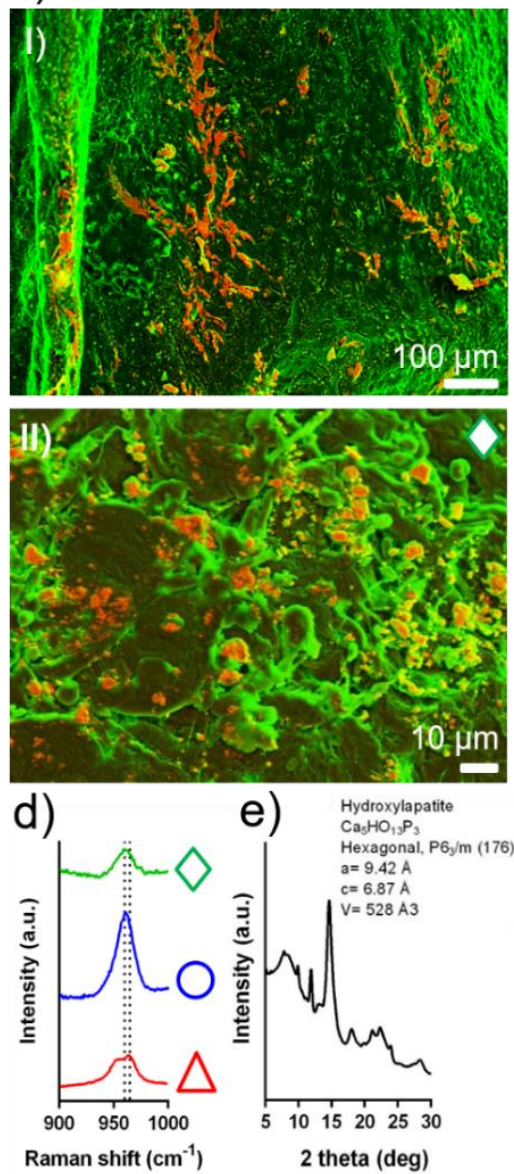

e) b) Villous region
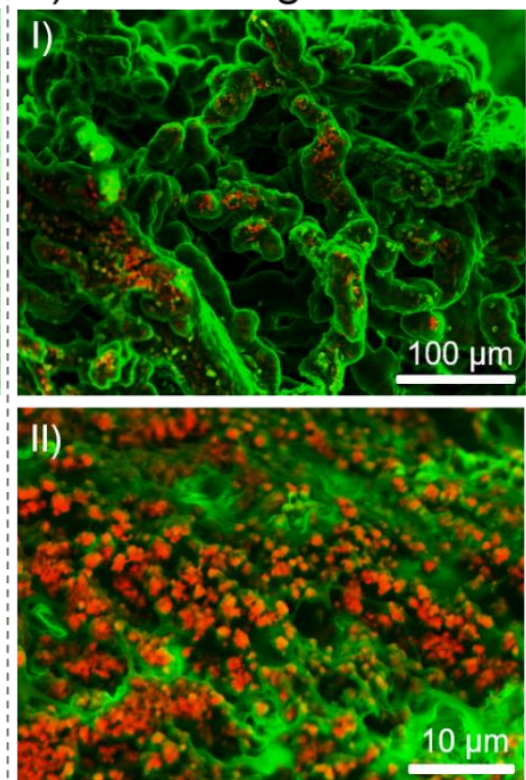
(6)

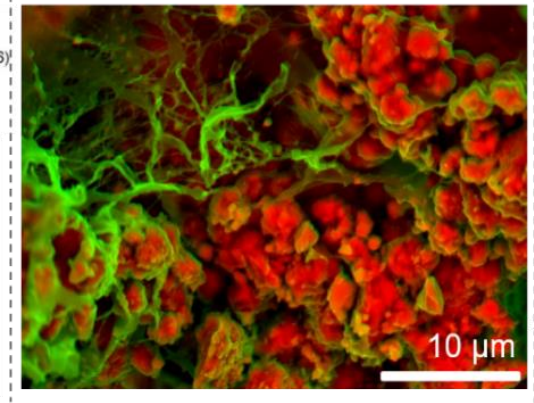

c) Maternal side
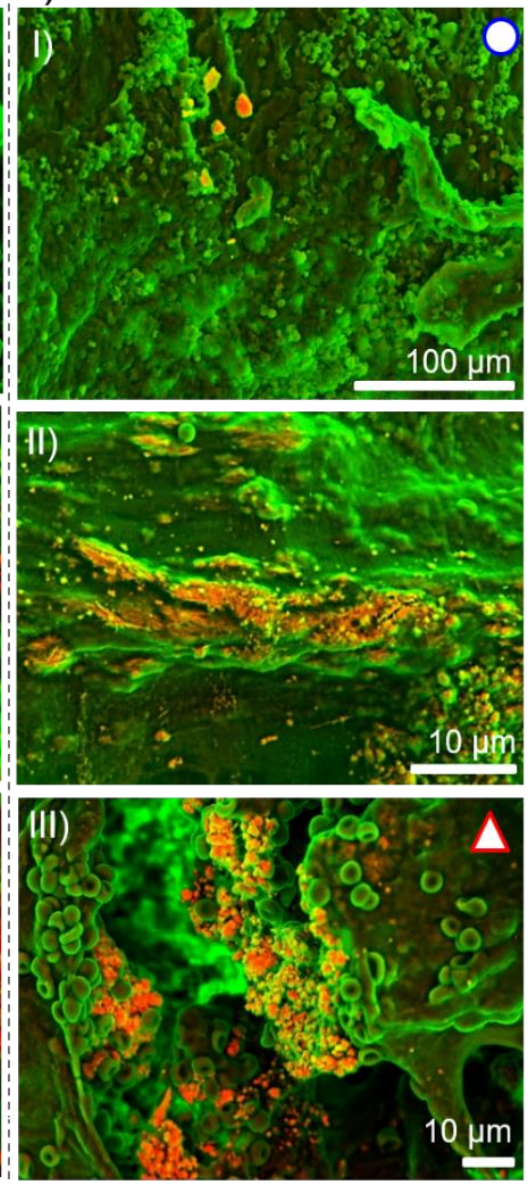

Figure 3: $(a-c)$ Density dependent color scanning electron micrographs (DDC-SEM) of placental tissue showing electron dense material (mineral) in red and organic material (tissue and cells) in green. Representative images show mineral structures found on (a) the chorial plate (fetal), (b) the villous region and (c) the basal plate (maternal side) at two different magnifications. (d) Corresponding Raman spectra indicating peak locations at 960 and $970 \mathrm{~cm}^{-1}$. (e) $X$-ray diffraction pattern of isolated mineral fraction extracted from placental tissue shows peaks characteristic for the hexagonal phase of hydroxyapatite. 